\title{
A Rare Case of Episodic Angioedema and Hypereosinophilia - Gleich Syndrome
}

\author{
Sharat Kumar B Jaikar ${ }^{1}$, Divya NS ${ }^{2}$ \\ ${ }^{I}$ (Department of Medicine, DM- Wayanad Institute of Medical Sciences, Kerala, India) \\ ${ }^{2}$ (Department of Pathology, DM- Wayanad Institute of Medical Sciences, Kerala, India)
}

\begin{abstract}
Gleich syndrome is a distinct clinical entity characterized by episodic angioedema, hypereosinophilia and increased body weight. Being a rare cause of hypereosinophilia, extensive diagnostic evaluation has to be carried out to rule out the other common causes like allergic diseases, Idiopathic hypereosinophliic syndrome (HES), connective tissue disorders, parasitic infestations, lympho-proliferative disorders and neoplastic conditions. We report a rare case of Gleich syndrome in a young Indian female. It usually runs a benign course and shows a good response to low dose corticosteroid therapy.
\end{abstract}

Keywords: Episodic angioedema, Gleich syndrome, hypereosinophilia, interleukin-5 (IL-5), recurrent urticaria, weight gain.

\section{Introduction}

Allergic diseases and parasitic infestation remains the major cause of hpereosinophilia in a subtropical region like India. Gleich syndrome is a one of the rare cause of hypereosinophilia associated with episodic angioedema, urticaria and periodic weight gain ${ }^{[1]}$. It was first described by Gerald Gleich ${ }^{[2]}$ in 1984, and since then several cases have been reported in Japan and South- East Asia ${ }^{[3]}$. Till date no cases have been documented in India due to under-recognition and rarity of presentation. Here, we report a rare case of this syndrome presenting with recurrent angioedema, hypereosinophilia, and weight gain without any parenchymal involvement. A dramatic response was observed with low dose corticosteroid treatment with normalization of eosinophil count.

\section{Case Report}

A 32 year old female presented with a recurrent history of angioedema and periodic weight gain for last two years. She had also experienced cyclical attacks of urticaria, fever, severe edema involving face, hands and legs. The attacks had occurred approximately once per month which lasted for about one week. Her past and family history was negative for hereditary angioedema, autoimmune diseases, allergic disorders and other common eosinophilic diseases.

On physical examination, non-pitting edema of face, lips, both the legs and hands was evident [Fig 1]. Faint erythemas of varying sizes were present on both the legs. Her body temperature was raised and other vital organs were within normal limits. Laboratory investigations revealed a total leukocyte count of $11,500 / \mathrm{mm}^{3}$ with raised eosinophil count (42\%). Haemoglobin and platelet counts were normal. Other investigations including immunological tests for connective tissue diseases, parasitology, blood chemistry, chest X-ray, CT scan of abdomen and pelvis were within normal limits. A course of anti-histaminics and Non-steroidal antiinflammatory drugs (NSAIDS) was prescribed. 15 days later patient returned back with similar complaints. Her weight was increased by 8 Kilograms and a severe degree of angioedema was noted.

Repeat investigations revealed $36 \%$ of eosinophils, hpereosinophilia on peripheral blood smear examination [Fig 2]. Bone marrow aspiration and biopsy was done to rule out any associated malignancy. Marrow study was normal except for mild increase in eosinophilic precursors and no atypical cells were seen. Other tests like urine analysis, serum electrophoresis, anti-nuclear antibodies (ANA), C-reactive protein and serum immunoglobulins like $\mathrm{IgG}, \operatorname{Ig} \mathrm{A}, \mathrm{IgE}, \mathrm{C}_{3}, \mathrm{C}_{4}$ and $\mathrm{C}_{1}$ Esterase levels were within normal limits. Her serum IgM levels was increased i.e., $496 \mathrm{mg} / \mathrm{dl}$ (normal range, $60-300 \mathrm{mg} / \mathrm{dl}$ ). A clinical diagnosis of hypereosnophilia with episodic angioedema - Gleich Syndrome was offered and patient was put on low dose corticosteroid therapy and vital signs were monitored periodically. All her symptoms got resolved with the normalization of eosinophil counts by 2 days (Fig 3). Patient was discharged and a regular follow up was advised with eosinophil count. She was asked to report back if the symptoms recur.

\section{Discussion}

Several cases of Gleich's syndrome have been described worldwide with variations in clinical presentations and laboratory findings ${ }^{[3]}$. The original cases decribed by Gleich et al ${ }^{[2]}$, presented with recurrent attacks of angioedema, urticaria, weight gain (10-18\%), hypereosinophilia and raised serum IgM levels, which 
was similar to our case. Over 10 years of surveillance, it showed a benign course without any parenchmal involvement and a good response to corticosteroid therapy. A few cases of hypereosinophila associated with non-episodic angioedema and normal IgM level was described in Japanese patients ${ }^{[4]}$. A new variant of this syndrome associated with eosinophilic ascites has also been documented ${ }^{[5]}$. Till date, ours is the first case to document this syndrome in India.

The exact etio-pathogenesis remains unclear. It is hypothesized that activated T-cells causes degranulation of eosinophils, releasing eosinophil major basic protein leading to edema, connective tissue damage and cutaneous lesions. Also involvement of IL-5 \& IL-2 has been strongly speculated in causing pruritis, angioedema, fever, weight gain, eosinophilia and erythematous rashes ${ }^{[6,7]}$. Few studies showed the presence of anti-endothelial cell antibodies, suggesting a role in increased capillary permeability with adhesion of eosinophils to vascular wall ${ }^{[8]}$.

For definitive diagnosis of Gleich syndrome, extensive laboratory evaluations are to be carried out to rule out allergic diseases, parasitic infestations, connective tissue disorders and neoplastic conditions. The characteristic features in our case include a young female presenting with recurrent attacks of angioedema, weight gain, hypereosinophilia, raised serum IgM level without involving vital organs like kidney, heart, lungs and liver. The underlying disorders causing edema, allergy, parasites, connective tissue diseases and malignancy were ruled out in our case. Other differential diagnosis related to hypereosinophilia may be considered. The criteria for Primary Hypereosiophilic Syndrome, includes persistent eosinophilia of $>1,500 / \mathrm{mm}^{3}$, for more than 6 months with systemic involvement ${ }^{[9]}$. In contrast, there will be transient eosinophilia in Gleich syndrome without any systemic involvement and usually carries a good prognosis.

Eosinophilia- myalgia syndrome is characterized by chronic fatigue, myalgia, paresthesia, respiratory symptoms and non-specific cutaneous involvement, which clearly distinguishes it from Gleich syndrome ${ }^{[10]}$. In capillary leak syndrome, there will be episodic edema, weight gain, but there is no eosinophilia and it is usually associated with renal failure and dysglobulemia ${ }^{[11]}$, whereas in Gleich syndrome parenchymal involvement is not seen and hypereosinophilia remains the major criteria.

The overall prognosis of Gleich Syndrome with low dose corticosteroids is good and the clinical flare ups are managed conservatively ${ }^{[7]}$.

\section{Figures}

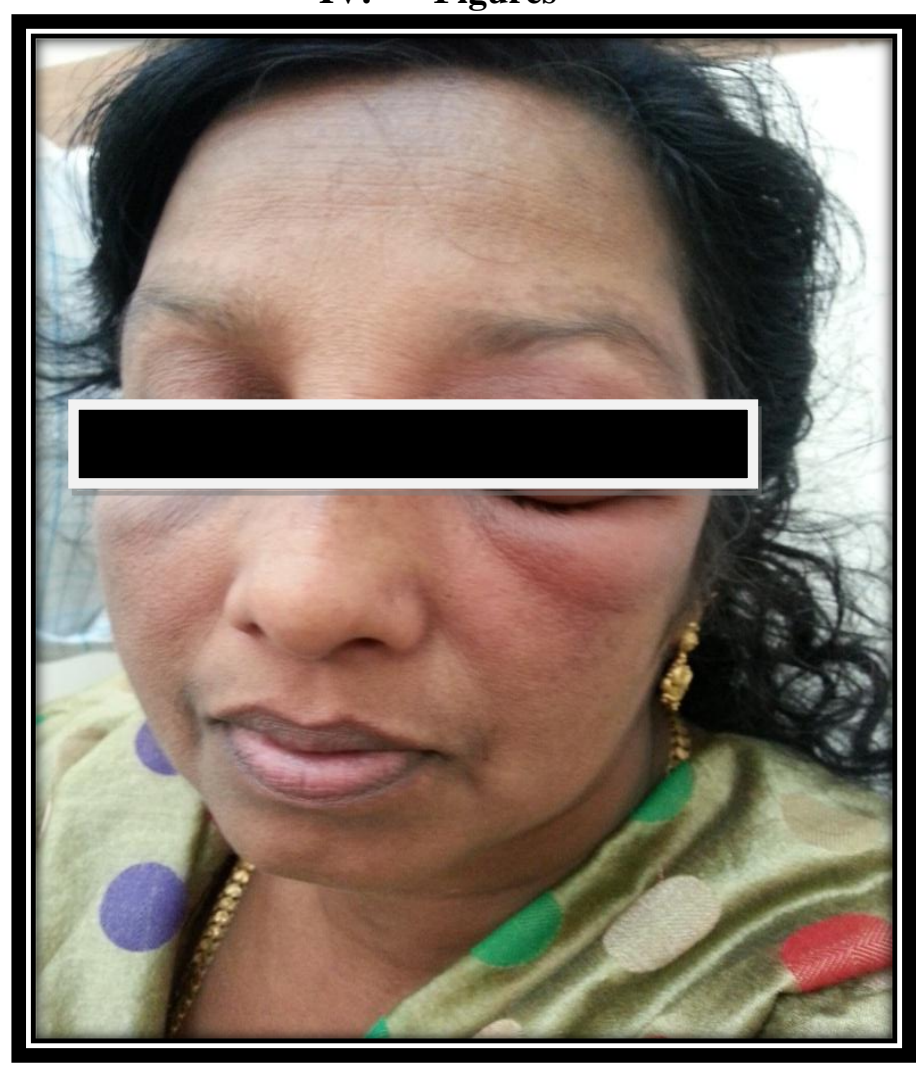

Fig 1: photograph of the patient showing severe angioedema of the face before treatment 


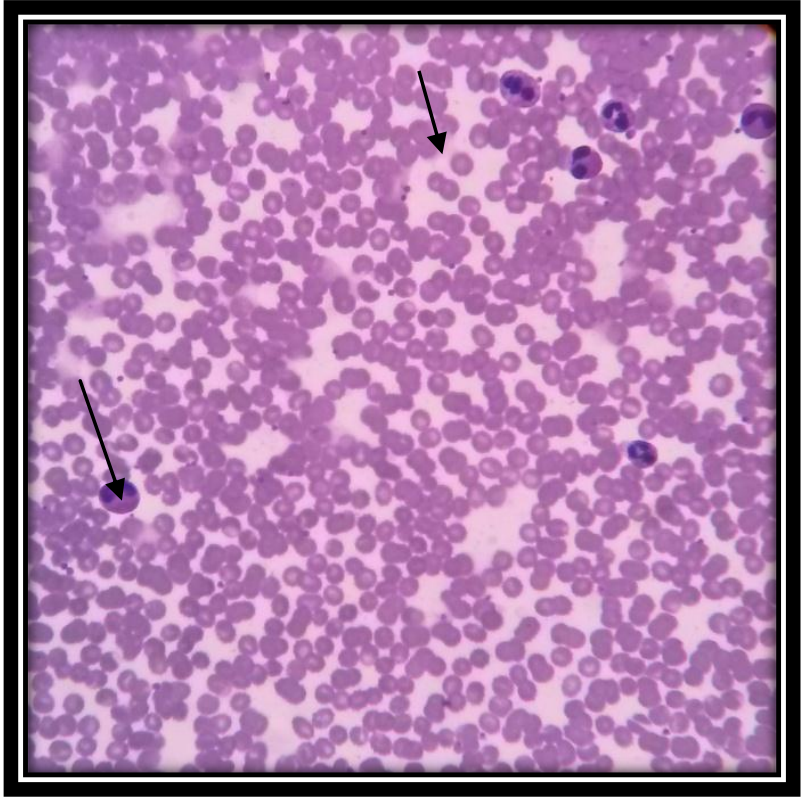

Fig. 2: Photomicrograph of peripheral blood smear showing increased eosinophils

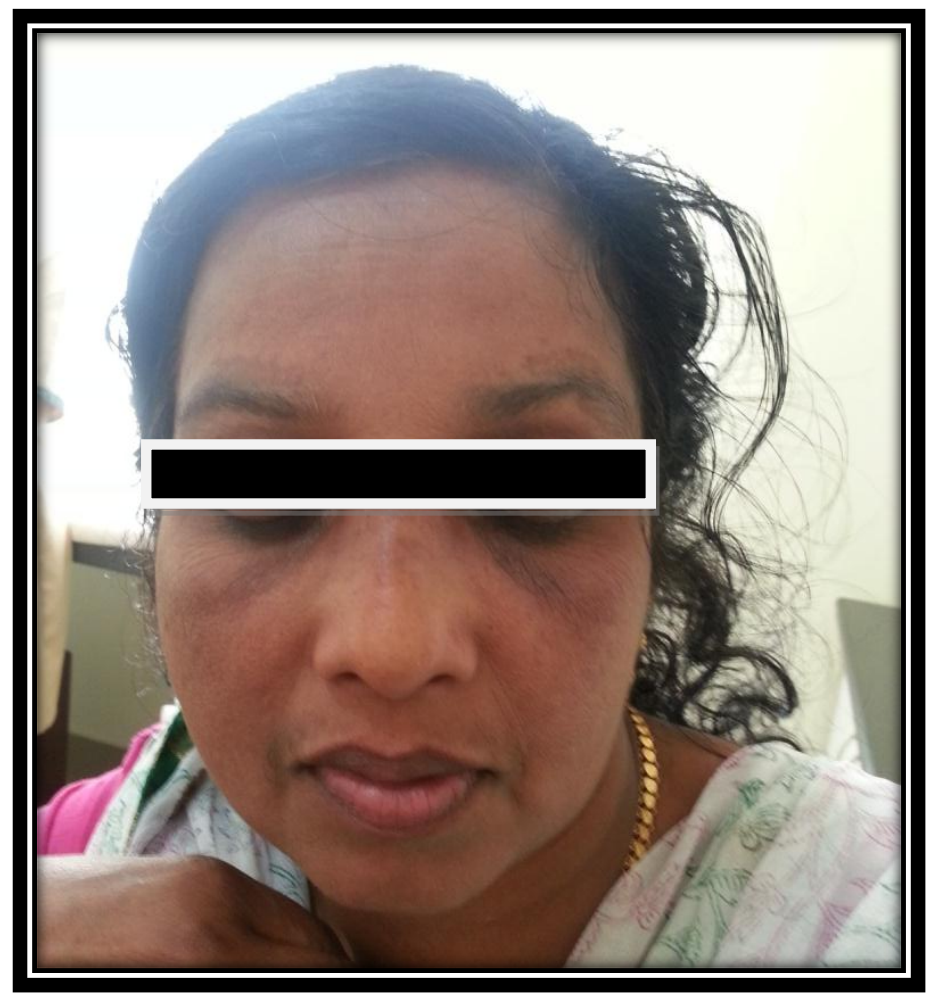

Fig 3: Photograph of patient showing decreased facial edema after treatment

\section{Conclusion}

A rare case of episodic angioedema and hypereosinophilia has been documented in young Indian women. Extensive diagnosic work up should be carried out to rule out the other possible causes of hypereosinophilia. We suggest that this rare clinical entity has to be widely recognized and documented for the appropriate and prompt management of the disease. 


\section{References}

[1] Abouzahir A, Chaurin P, Coutant G and Garcin JM, Gleich syndrome- A case report and review of the literature, Rev Med Interne, 26(2), 2005, 137-40.

[2] Gleich GJ, Schroeter AL, Marcoux P, Sachs MI, O’Connel EJ and Kohler PF, Episodic angioedema associated with eosinophilia, N Engl J Med, 310, 1984, 1621-6.

[3] Takanori S, Yoshio U, Hirotsugu T and Seiji A, A case of episodic angioedema associated with eosinophilia, The J of Med. Investigation, 44, 1997, 103.

[4] Shimasaki AK, Five cases of nonepisodic angioedema with eosinophilia, Rinsho Ketsueki, 42(8), 2001, 639-43.

[5] Nasser RD, Sajid M, Khalid K, Amjad S and Shahid M, Recurrent urticaria, blood eosinophilia and eosinophilic ascites- A new variant of Gleich's syndrome, J of Army Medical Corps, 3, 2008, 1-4.

[6] Rosenberg SA, Lotze MT, M uul LM, Observations on the systemic administration of autologous lymphokine activated killer cells and recombinant interleukin-2 to patients with metastatic cancer, N Engl J Med, 313, 1985, 1485.

[7] JH Butterfield, KM Leiferman, J Abrams, JE Silver and J Bower, Elevated levels of IL-5 in patients with the syndrome of episodic angioedema and eosinophilia, blood,79(3), 1992, 688-92.

[8] Lassalle P, Gosset P, Gruart V, Prin L, Capron M and Lagrue G, Presence of antibodies against endothelial clls in the sera of patients with episodic angioedema and hypereosinophilia, Clin Exp Immunol,82,1990,38-43.

[9] Chiharu T, Tadahiro K, Kyoji I and Yoshiji Y, Four Japanese cases of episodic angioedma with eosinophilia, Internal Med, 31(4), 1992, 470-73.

[10] Kaufmann L, Krupp LB, Eosinophilia- myalgia syndrome, toxic-oil syndrome, and diffuse fasciitis with eosinophilia, Curr Opin Rheumatol,7, 1995, 560-7.

[11] Bonadies N, Baud P, Peter HJ, Buergi U and Mueller BU, A case report of Clarkson's disease- if you don't know it, you will miss it, Eur J Intern Med,17(5), 2006, 363-5. 